\title{
ON A THEOREM OF POSNER
}

\author{
MANSOOR AHMAD
}

\begin{abstract}
Posner [1] has proved the following theorem: Let $R$ be a prime ring ${ }^{1}$ and $d a$ derivation of $R$ such that, for all $a \in R$, ada - daa $\in Z$ (centre of $R$ ). Then, if $d$ is not a zero derivation, $R$ is commutative.
\end{abstract}

Two proofs of this theorem are known-one by Posner himself and another by Ram Awtar [2]. It is natural to expect that the proof would be short and simple in the case when $R$ is of characteristic 2, but, by chance, these proofs are somewhat contrary to this expectation. Although, in this case, the condition $a d a-d a a \in Z$ is the same as $d\left(a^{2}\right) \in Z$, which implies $d(a b+b a) \in$ $Z$ for all $a, b \in R$.

The object of this paper is to give a very simple, short and direct proof of this theorem in the case when $R$ is of characteristic 2 , then to generalize this technique to prove a lemma which is similar to the result that, if $d(a b-b a)$ $\in Z$, when $R$ is not necessarily of characteristic 2 , then either $d$ is zero or $R$ is commutative, and lastly to prove a generalized form of the main theorem, i.e. Theorem 1 of Awtar [3], which follows as a consequence of the lemma.

Proof of PoSNER'S THEOREM, IN THE CASE WHEN $R$ IS OF CHARACTERISTIC 2 . The condition $a d a-d a a \in Z$ is the same as $d\left(a^{2}\right) \in Z$, which gives $d(a b+b a) \in Z$ if we replace $a$ by $(a+b)$. Commuting $d(a b+b a)$ with $a$ we have

$$
a d(a b+b a)=d(a b+b a) a .
$$

Replacing $b$ by $b a$ in (i) and using (i), we have

$$
a(a b+b a) d a=(a b+b a) d a a .
$$

Replacing $b$ by $d a$ in (ii), since $a d a-d a a \in Z$, and $R$ is of characteristic 2 , we have

$$
(a d a-d a a)^{2}=0 .
$$

Since $R$ is prime, this implies $a d a-d a a=0$. Consequently, we have

$$
d(a b+b a)=0 .
$$

Replacing $b$ by $b a$, we have

$$
(a b+b a) d a=0 .
$$

Received by the editors July 7, 1976.

AMS (MOS) subject classifications (1970). Primary 16A66, 16A72; Secondary 16A48.

Key words and phrases. Prime rings and derivation.

${ }^{1}$ All rings considered in this paper are associative. For definitions see [4].

- American Mathematical Society 1977 
Replacing $b$ by $b c, c \in R$, in the last result and using the last result, since $R$ is prime, it follows that either $d$ is zero or $R$ is commutative.

LemMa. Let $R$ be a prime ring of characteristic not 2. Let $S$ be the subgroup of the additive group of $R$ generated by all $A B-B A$ and $(A B-B A) A$, where $A=x y-y x, B=a b-b a$, and $a, b, x, y$ run through $R$. If $d$ is a derivation of $R$ such that $d V \in Z$ (centre of $R$ ) for all $V \in S$, then $d$ is zero or $R$ is commutative.

Proof. Since $d\left(A_{1} B_{1}-B_{1} A_{1}\right) \in Z$, choosing $A_{1}=V=A B-B A$ or $(A B$ - $B A) A, B_{1}=V x-x V, x \in R$, we have $d[V(V x-x V)-(V x-x V) V]$ $\in Z$. Since $d V \in Z$, it follows easily that $p^{2}(d x) \in Z$, where $p(x)=V x-$ $x V$. Replacing $x$ by $x V$ in this result, we have

$$
p^{2}(d x) V+p^{2}(x) d V \in Z
$$

Putting $d x$ for $x$ in (I), and using the results $d V \in Z, p^{2}(d x) \in Z$, we have

$$
p^{2}\left(d^{2} x\right) V \in Z \text {. }
$$

Since $p^{2}\left(d^{2} x\right) \in Z$ and $R$ is prime, either $p^{2}\left(d^{2} x\right)=0$ or $V \in Z$. If $p^{2}\left(d^{2} x\right)=$ 0 , replacing $x$ by $x V$ in this result, we have

$$
p^{2}\left(d^{2} x V+2 d x d V+x d^{2} V\right)=0
$$

i.e.

$$
2 p^{2}(d x) d V+p^{2}(x) d^{2} V=0
$$

because $x d V-d V x=0$, and so $d(x d V-d V x)=0$ or $x d^{2} V-d^{2} V x=0$, i.e. $d^{2} V \in Z$. Since $d V \in Z$ and $p^{2}(d x) \in Z$, (III) implies $p^{2}(x) d^{2} V \in Z$. Since $R$ is prime, either $d^{2} V=0$ or $p^{2}(x) \in Z$. If $d^{2} V=0$, since $R$ is not of characteristic 2 , by (III), we have

$$
p^{2}(d x) d V=0
$$

Since $d V \in Z$ and $R$ is prime, we have $d V=0$ or $p^{2}(d x)=0$. If $p^{2}(d x)=0$, by (I), we have $p^{2}(x) d V \in Z$, so either $d V=0$ or $p^{2}(x) \in Z$. If $p^{2}(x) \in Z$, replacing $x$ by $x V$, we have $p^{2}(x) V \in Z$. Therefore, either $p^{2}(x)=0$ or $V \in Z$. If $p^{2}(x)=0$, replacing $x$ by $x y, y \in R$, it follows easily that $V \in Z$, and since $V=A B-B A$ or $(A B-B A) A$, we have $A B-B A=0$, i.e. $V=$ 0 . Hence in each case $d V=0$.

Since $d V=0$, we have $d(A B-B A)=0$ and $d[(A B-B A) A]=0$. Therefore, we have

$$
(A B-B A) d A=0 .
$$

Replacing $B$ by $V_{1}$ in (IV), where $V_{1}=A B_{1}-B_{1} A$, and using (IV), we have

$$
\left(A B_{1}-B_{1} A\right) A d A=0 .
$$

Again, replacing $B_{1}$ by $A B_{2}-B_{2} A$ in (V), and using (V), we have

$$
\left(A B_{2}-B_{2} A\right) A^{2} d A=0 .
$$

Therefore, it follows that 


$$
V d A=0 \text { and } V A d A=0 \text {, }
$$

for all $V \in S$. Replacing $B$ by $V x-x V$ in (IV), $V \in S, x \in R$, and using (VI), we have

$$
(A V x-V x A) d A=0 .
$$

Replacing $x$ by $x B_{1}$ in (VII), where $B_{1}=z y-y z, y, z \in R$, we have

$$
\left[(A V x-V x A) B_{1}+V x\left(A B_{1}-B_{1} A\right)\right] d A=0 .
$$

By (IV), with $B_{1}$ for $B$, the second term on the left-hand side vanishes, so we have

$$
(A V x-V x A)(z y-y z) d A=0 .
$$

Replacing $A V x-V x A$ by $H$ and $y$ by $H y$ in (VIII), we have

$$
H[(z H-H z) y+H(z y-y z)] d A=0 .
$$

By (VIII), the second term on the left-hand side vanishes, and since $R$ is prime, we have

$$
H(z H-H z)=0 \text { or } \quad d A=0 .
$$

Therefore, either $H \in Z$ or $d A=0$. If $H \in Z$, by (VII), it follows that either $H=0$ or $d A=0$. If $H=0$, i.e. if $A V x-V x A=0$, replacing $x$ by $S A t, S$, $t \in R$, and using $H=0$, with $x$ replaced by $S$, we have

$$
V S A(A t-t A)=0 \text {. }
$$

Therefore, either $V=0$ or $A \in Z$. If $V=0$, then $A$ commutes with $B$, i.e. $A$ commutes with $(x y-y x), x, y \in R$. Replacing $y$ by $y x$, it follows that $A$ commutes with $(x y-y x) x$, so we have

$$
(x y-y x)(x A-A x)=0 .
$$

Therefore, replacing $y$ by $y z, z \in R$, it follows that $A \in Z$, i.e. $x y-y x \in Z$, so $(x y-y x) x \in Z$, which implies that $R$ is commutative. If $A \in Z$, then $A$ commutes with $B$, and it follows, as before, that $R$ is commutative. If $d A=0$, we have $d(x y-y x)=0, x, y \in R$. Replacing $y$ by $y x$, we have $(x y-y x) d x$ $=0$. Replacing $y$ by $y z, z \in R$, it follows that either $d$ is zero or $R$ is commutative. This completes the proof of the lemma.

THEOREM. Let $T(S)$ be a subgroup of the additive group of $R$, where $R$ and $S$ are the same as before, such that $V \in T(S)$ and $V t-t V \in T(S)$, for every $V$ and $t, V \in S, t \in R$. Let us suppose that $R$ is not of characteristic 2 or 3 , and let $d$ be a nonzero derivation of $R$, with $U d U-d U U \in Z$ (centre of $R$ ) for all $U \in T(S)$. Then $R$ is commutative.

Proof. Since by hypothesis $V \in T(S)$ and $V r-r V \in T(S)$, for all $V$ and $r, V \in S, r \in R$, replacing $u$ by $V$ in Lemma 2 of Ram Awtar [2], it follows that $[[d r, V], V] \in Z$, where $[x, y]=x y-y x, x, y \in R$. Now, proceeding on the same lines as in Posner [1] (cf. equations (16) to (27)), we have $[V, d V]=0$, for all $V \in S$. Putting $r V$ for $r$ in $p^{2}(d r) \in Z$, where $p(r)=V r$ $-r V$, and using 


$$
p^{2}(x y)=p^{2}(x) y+2 p(x) p(y)+x p^{2}(y), \quad x, y \in R,
$$

we have

$$
p^{2}(d r) V+p^{2}(r) d V \in Z
$$

Commuting this with $V$ and using $[V, d V]=0, p^{2}(d r) \in Z$, we have

$$
\left[V, p^{2}(r)\right] d V=0,
$$

i.e. $p^{3}(r) d V=0$. Putting $y r$ for $r, y \in R$, and using

$$
p^{3}(y r)=p^{3}(y) r+3 p^{2}(y) p(r)+3 p(y) p^{2}(r)+y p^{3}(r),
$$

we have

$$
\left\{p^{3}(y) r+3 p^{2}(y) p(r)+3 p(y) p^{2}(r)\right\} d V=0 .
$$

Putting $d y$ for $y$ and $p(r)$ for $r$, and using $p^{2}(d y) \in Z, p^{3}(d y)=0$, we have either $p^{2}(d y)=0$ or $p^{2}(r) d V=0$. If $p^{2}(r) d V=0$, then, since $p^{2}(d y) V+$ $p^{2}(r) d V \in Z$ and $p^{2}(d y) \in Z$, it follows that $p^{2}(d y)=0$. Replacing $y$ by $r V$, we have $p^{2}(r) d V=0$. Replacing $r$ by $y p(r)$, we have $p^{2}(y) p(r) d V=0$, and replacing $r$ by $p(y) r$ in $p^{2}(r) d V=0$, we have either $d V=0$ or $p^{3}(y)=0$. If $p^{3}(y)=0$, replacing $y$ by $y r$, we have $p^{2}(y) p(r)+p(y) p^{2}(r)=0$. Replacing $y$ by $d y$, we have $p(d y) p^{2}(r)=0$, and replacing $y$ by $p(y)$ in $p^{2}(y) p(r)+$ $p(y) p^{2}(r)=0$, we have $p^{2}(y) p^{2}(r)=0$. Replacing $y$ by $y d x, x \in R$, we have $p^{2}(y) d x p^{2}(r)=0$. Replacing $x$ by $\left(x p^{2}(t)\right) a, t \in R, a \in R$, we have either $p^{2}(r)=0$ and, consequently, $V \in Z$, or $d\left(p^{2}(t)\right)=0$, which reduces to $I_{d V} I_{V}(t)=0$, where $I_{a}$ denotes inner derivation by $a$, because $p^{2}(d t)=0$ and $V d V=d V V$. Consequently, by Posner [1, Theorem 1], it follows that $V \in Z$ or $d V \in Z$. If $V \in Z$, we have $V r-r V=0, r \in R$. Therefore, $d(V r-r V)$ $=0$, and we have $d V \in Z$, i.e. in each case $d V \in Z$ for all $V \in S$. Since $p^{2}(r) d V=0$, it follows that either $d V=0$ or $V \in Z$, for all $V \in S$. Hence, by the lemma, it follows that $R$ is commutative.

ACKNOWLEDGEMENT. The author takes pleasure in expressing his thanks to the referee for helpful comments and remarks.

\section{REFERENCES}

1. E. C. Posner, Derivation in prime rings, Proc. Amer. Math. Soc. 8 (1957), 1093-1100.

2. R. Awtar, On a theorem of Posner, Proc. Cambridge Philos. Soc. 73 (1973), 25-27.

3. (1973), 67-74.

4. I. N. Herstein, Topics in ring theory, Univ. of Chicago Press, Chicago, Ill., 1969.

AMINABAD, LuCKNow, INDIA 